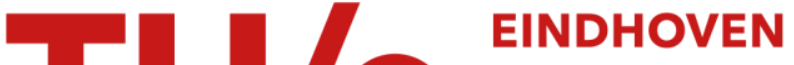 \\ UNIVERSITY OF \\ TECHNOLOGY
}

\section{Effects of exciton deconfinement on the transient photoluminescence from thermally activated delayed fluorescence host-guest systems}

Citation for published version (APA):

Hauenstein, C., Gottardi, S., Bobbert, P. A., Coehoorn, R., \& van Eersel, H. (2020). Effects of exciton deconfinement on the transient photoluminescence from thermally activated delayed fluorescence host-guest systems. Journal of Applied Physics, 128(7), [075501]. https://doi.org/10.1063/5.0019073

\section{Document license:}

TAVERNE

DOI:

$10.1063 / 5.0019073$

Document status and date:

Published: 21/08/2020

Document Version:

Publisher's PDF, also known as Version of Record (includes final page, issue and volume numbers)

Please check the document version of this publication:

- A submitted manuscript is the version of the article upon submission and before peer-review. There can be important differences between the submitted version and the official published version of record. People interested in the research are advised to contact the author for the final version of the publication, or visit the $\mathrm{DOI}$ to the publisher's website.

- The final author version and the galley proof are versions of the publication after peer review.

- The final published version features the final layout of the paper including the volume, issue and page numbers.

Link to publication

\footnotetext{
General rights

- You may freely distribute the URL identifying the publication in the public portal. follow below link for the End User Agreement:

www.tue.nl/taverne

Take down policy

If you believe that this document breaches copyright please contact us at:

openaccess@tue.nl

providing details and we will investigate your claim.
}

Copyright and moral rights for the publications made accessible in the public portal are retained by the authors and/or other copyright owners and it is a condition of accessing publications that users recognise and abide by the legal requirements associated with these rights.

- Users may download and print one copy of any publication from the public portal for the purpose of private study or research.

- You may not further distribute the material or use it for any profit-making activity or commercial gain

If the publication is distributed under the terms of Article 25fa of the Dutch Copyright Act, indicated by the "Taverne" license above, please 


\section{Effects of exciton deconfinement on the transient photoluminescence from thermally activated delayed fluorescence host-guest systems}

Cite as: J. Appl. Phys. 128, 075501 (2020); https://doi.org/10.1063/5.0019073

Submitted: 19 June 2020 . Accepted: 21 July 2020 . Published Online: 18 August 2020

C. Hauenstein, (D) S. Gottardi, (D) P. A. Bobbert, (iD) R. Coehoorn, and (i) H. van Eersel
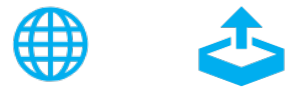

\section{ARTICLES YOU MAY BE INTERESTED IN}

Efficiency loss processes in hyperfluorescent OLEDs: A kinetic Monte Carlo study

Applied Physics Letters 114, 073301 (2019); https://doi.org/10.1063/1.5079642

Impact of secondary donor units on the excited-state properties and thermally activated delayed fluorescence (TADF) efficiency of pentacarbazole-benzonitrile emitters The Journal of Chemical Physics 153, 144708 (2020); https://doi.org/10.1063/5.0028227

A perspective on blue TADF materials based on carbazole-benzonitrile derivatives for efficient and stable OLEDs

Applied Physics Letters 116, 120503 (2020); https://doi.org/10.1063/1.5143501

\section{Challenge us.} What are your needs for periodic signal detection?

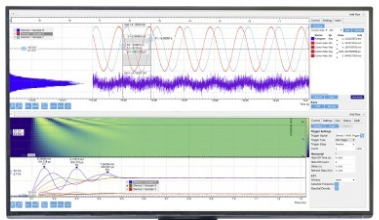

○
Zurich - Instruments 


\title{
Effects of exciton deconfinement on the transient photoluminescence from thermally activated delayed fluorescence host-guest systems
}

Cite as: J. Appl. Phys. 128, 075501 (2020); doi: 10.1063/5.0019073

Submitted: 19 June 2020 - Accepted: 21 July 2020 .

Published Online: 18 August 2020

C. Hauenstein, ${ }^{1,2, a)}$ (iD S. Gottardi, ${ }^{1}$ (D) P. A. Bobbert, ${ }^{2}$ (D) R. Coehoorn, ${ }^{2}$ (D) and H. van Eersel ${ }^{1}$ (iD)

\begin{abstract}
AFFILIATIONS
${ }^{1}$ Simbeyond B.V., Groene Loper 5, 5612 AE Eindhoven, The Netherlands

${ }^{2}$ Department of Applied Physics and Institute for Complex Molecular Systems, Eindhoven University of Technology,

P.O. Box 513, 5600 MB Eindhoven, The Netherlands
\end{abstract}

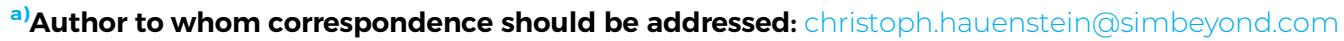

\begin{abstract}
For thermally activated delayed fluorescence (TADF) host-guest systems used in organic light-emitting diodes, understanding of the transient photoluminescence $(\mathrm{PL})$ measurements is crucial for accurate determination of the photophysical rates of the emitter. Here, we study how the PL is affected by triplet-exciton deconfinement from the guest to the host molecules. This deconfinement can complicate the analysis of the PL decay and potentially lead to a loss of efficiency. From an analytical model, we find that the transient PL intensity remains bi-exponential in the presence of exciton deconfinement for the case of fast triplet diffusion, albeit with a longer decay time of the delayed component. Deconfinement might, therefore, not always be recognizable from a single transient PL measurement. The role of deconfinement depends on the energetic disorder, the guest concentration, and the energy difference $\Delta E_{\mathrm{T}}$ between triplet-exciton energies on the host and guest molecules and is effectively suppressed for $\Delta E_{\mathrm{T}}>0.2 \mathrm{eV}$. We find from analytical modeling and kinetic Monte Carlo simulations that the decay can become non-bi-exponential and even show a distinct third decay step. The shape of the decay curves depends on the characteristic times for guest-host transfer and host diffusion, relative to the prompt and delayed decay times of the TADF emitter. A comparison with available experimental data is included, finding qualitative agreement with dedicated deconfinement studies and indicating the influence of other processes for the often observed power-law decay at long time scales.
\end{abstract}

\section{Published under license by AIP Publishing. https://doi.org/10.1063/5.0019073}

\section{INTRODUCTION}

At low luminance levels, the internal quantum efficiency (IQE) of organic light-emitting diodes (OLEDs) that utilize thermally activated delayed fluorescence (TADF) can be close to $100 \% .{ }^{1-3}$ These high efficiencies are obtained by using fluorescent emitter molecules that also have a very small energy gap between the singlet and triplet states. This allows efficient thermally activated conversion of triplet excitons to the singlet state by reverse intersystem crossing (rISC; see Fig. 1). Most commonly, the rates of the relevant intramolecular photophysical processes are deduced from time-resolved photoluminescence (PL) and total PL yield experiments, assuming a simple three-level model that includes the $\mathrm{S}_{0}$ singlet ground state, the $\mathrm{S}_{1}$ lowest singlet excited state, and the $\mathrm{T}_{1}$ lowest triplet excited state. The time $(t)$-dependent PL intensity
$I_{\mathrm{PL}}(t)$ may then be expressed as a sum of an exponentially decreasing prompt and delayed contribution. Various schemes have been developed for deducing the photophysical rates (black full and dashed arrows in the left-hand part of Fig. 1) from measured $I_{\mathrm{PL}}(t)$ curves. $^{4-8}$ Accurate knowledge of these rates is necessary to evaluate candidates for emitter materials, for understanding the IQE at low luminance levels, and for developing a mechanistic device model that also includes the bimolecular loss processes (excitonpolaron quenching and exciton-exciton annihilation) that give rise to a decrease of the IQE ("roll-off") with increasing luminance."

The processes determining the time-resolved PL intensity decay can actually be more complicated than that sketched above. First, for some TADF emitters, additional intramolecular excited states are involved. These can be higher-energy triplet $\left(T_{n}\right)$ states within a manifold of states with different degrees of localized and 


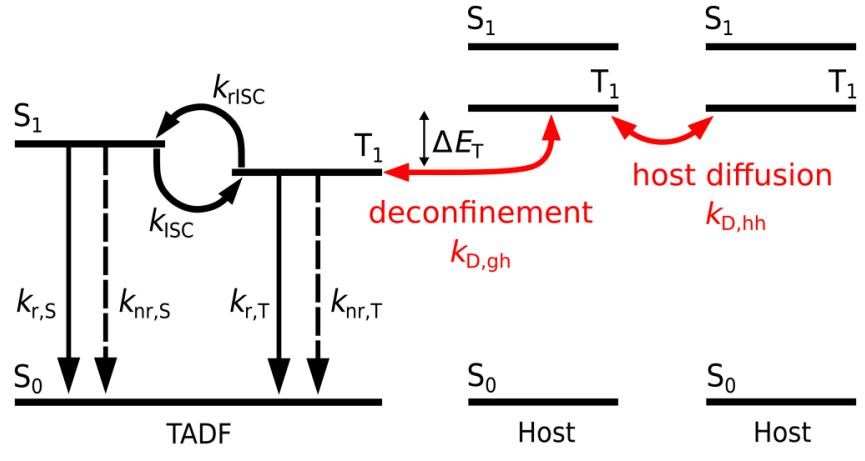

FIG. 1. Photophysical and exciton transfer rates in the host-guest systems studied in this work. The photophysical rates of the TADF emitter molecules are described assuming a three-level model, with (non-)radiative singlettriplet decay rates $k_{(\mathrm{n}) \mathrm{r}, \mathrm{S} / \mathrm{T}}$ and (reverse) intersystem crossing rates $k_{(\mathrm{r}) \mid \mathrm{Sc}}$. The red arrows indicate the Dexter transfer of triplets to the host material and the subsequent diffusion over the host. The distance $(R)$ and energy difference $(\Delta E)$ dependencies of the guest-host and host-host Dexter transfer rates $k_{D, g h}(R, \Delta E)$ and $k_{\mathrm{D}, \mathrm{hh}}(R, \Delta E)$, respectively, are given in Sec. $\| C . \Delta E_{\mathrm{T}}$ is the triplet deconfinement energy barrier.

charge-transfer (CT) wavefunction character, and also higherenergy singlet states with a mixed localized and CT character. ${ }^{10-14}$ Second, in many TADF OLEDs, the TADF emitter molecules are embedded at a low concentration in a host material, in order to avoid efficiency losses due to, e.g., concentration quenching. In such systems, interactions with the host can further complicate the analysis of the time-resolved PL intensity decay and can obscure the role of more complex intramolecular processes. Examples of such effects are (i) varying energy level shifts, in some cases even time-dependent, due to dielectric screening of excitations on the TADF molecules by the polarizable environment, (ii) the formation of CT-states involving host molecules, and (iii) exciton transfer to states fully located on the host molecules ("deconfinement"). ${ }^{15-18}$

In this paper, we study the effect of exciton deconfinement on the transient PL intensity. For TADF host-guest systems, the critical confinement energy is that of the triplet excitons, $\Delta E_{\mathrm{T}} \equiv E_{\mathrm{T}, \mathrm{h}}-E_{\mathrm{T}, \mathrm{g}}$. Here, $E_{\mathrm{T}, \mathrm{h}}$ and $E_{\mathrm{T}, \mathrm{g}}$ are the host and guest triplet energies, respectively. As the host usually has a relatively large singlet-triplet gap, singlet excitons on the TADF guest are generally well-confined. The right-hand side of Fig. 1 shows in a schematic manner the processes that are included: triplet transfer from guest to host molecules (the direct deconfinement process) and triplet transfer between host molecules (host diffusion, which accelerates the deconfinement process). Different deconfinement regimes are identified and an analytical model is used where applicable. Due to the complex interplay of the different processes, some regimes have to be studied using three-dimensional (3D) kinetic Monte Carlo (KMC) simulations. In this work, we focus on the sensitivity to the confinement energy, the exciton diffusivity on the host, the TADF emitter concentration, and the energetic disorder of the host triplet states.

In OLEDs, diffusion of deconfined triplet excitons over the host can give rise to additional losses, e.g., due to quenching at defects, and enhanced triplet-polaron quenching and triplet-exciton annihilation. ${ }^{17,19}$ Our study leads to design rules for the host triplet energy, which should be such that on the one hand deconfinement is minimized, whereas, on the other hand, the host material should not have an unnecessarily large energy gap in order to avoid large overvoltages.

The paper is organized as follows. In Sec. II, we develop exact solutions of the differential equations that describe the photoluminescence $I_{\mathrm{PL}}(t)$ and deconfinement processes for two situations: (i) very fast guest-host transfer and very fast diffusion over the host and (ii) no diffusion over the host, but deconfinement due to guest-host transfer. In addition, we analyze using continuum diffusion theory the "intermediate host-diffusion" regime, within which deconfinement due to host diffusion occurs entirely in the delayed emission regime. In Sec. III, we use 3D-KMC simulations to study triplet deconfinement for a wide range of parameters that we consider as realistic for TADF OLEDs, making use of the results of a recent study of triplet-exciton diffusion over various hosts. ${ }^{20} \mathrm{We}$ discuss the effects of varying the host diffusion coefficient, the guest-host transfer rate, the guest concentration, and the photophysical rates of the guest molecules and show under which conditions the fast-diffusion and no-diffusion limits that were discussed in Sec. II are approached. Section IV contains a summary and conclusions.

\section{THE FAST, INTERMEDIATE, AND ZERO-HOST-DIFFUSION REGIMES}

Triplet deconfinement affects the time-resolved delayed PL intensity due to the resulting reduced density of triplet excitons on the guest molecules. In this section, we investigate the effect for three regimes. First, we consider very fast guest-host deconfinement and very fast host diffusion. In that limit, thermodynamic equilibrium is established between the excitons on the host and guest molecules, so that the reduction of the density of triplet excitons on the guest molecules follows from Boltzmann statistics. Second, we discuss the effect of deconfinement for the specific case of equal but finite guest-host and host-host transfer rates. Third, we analyze the effect of deconfinement in the absence of host diffusion, where transfer can only occur from a guest to a host site and back. Throughout this paper, we consider host and guest triplet states with a Gaussian density of states with widths (standard deviations) $\sigma_{\mathrm{h}}$ and $\sigma_{\mathrm{g}}$, respectively. The analytical expressions that are developed in this section will support the interpretation of the results from KMC simulations for more general situations, presented in Sec. III.

\section{A. The fast-diffusion limit}

In the limit of fast guest-host triplet-exciton transfer and fast diffusion over the host ("fast-diffusion limit"), the probability that a triplet in the system resides on a guest molecule follows from Boltzmann statistics and is given by

$$
P_{\mathrm{T}, \mathrm{g}}=\frac{c_{\mathrm{g}}}{c_{\mathrm{g}}+c_{\mathrm{h}} \exp \left[-\frac{\Delta E_{\mathrm{T}}}{k_{\mathrm{B}} T}+\frac{\sigma_{\mathrm{h}}^{2}-\sigma_{\mathrm{g}}^{2}}{2\left(k_{\mathrm{B}} T\right)^{2}}\right]},
$$




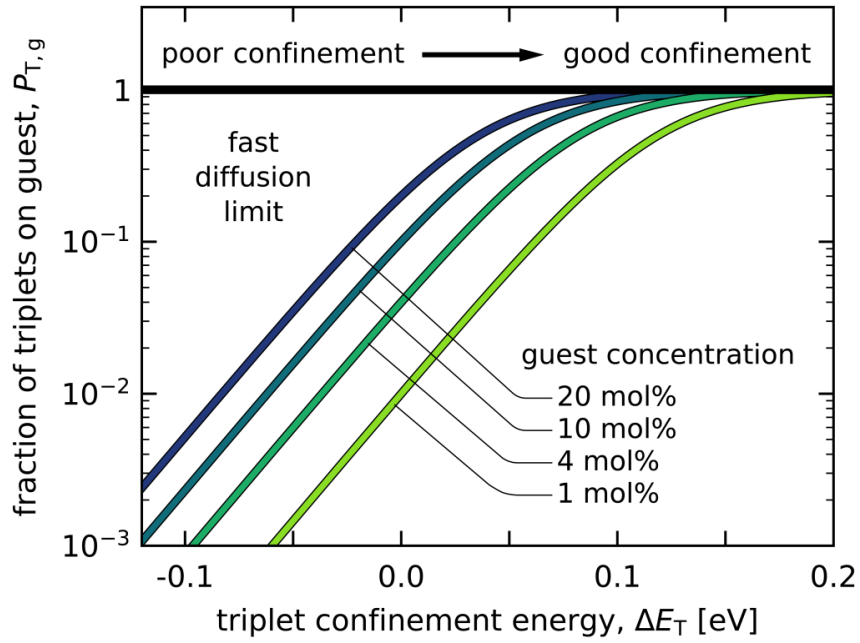

FIG. 2. Dependence of the fraction of triplet excitons residing on guest molecules $P_{\mathrm{T}, g}$ on the triplet confinement energy $\Delta E_{\mathrm{T}}$ and on the emitter (guest) concentration, in the limit of fast triplet-exciton diffusion over the host and assuming equal host and guest energy disorder widths $\left(\sigma_{\mathrm{h}}=\sigma_{\mathrm{g}}\right)$.

where $c_{\mathrm{h}}$ and $c_{\mathrm{g}} \equiv\left(1-c_{\mathrm{h}}\right)$ are the host and guest concentrations, respectively, $T$ is the temperature, and $k_{\mathrm{B}}$ is the Boltzmann constant. Figure 2 shows the dependence of $P_{\mathrm{T}, \mathrm{g}}$ on $\Delta E_{\mathrm{T}}$ and on the guest concentration, for equal host and guest disorder widths. The figure shows that in the fast-diffusion limit, depending on the guest concentration, $\Delta E_{\mathrm{T}}$ of at least $0.1-0.2 \mathrm{eV}$ is required to ensure the excitons still spend a large fraction of time on the guest sites. The limit of fast diffusion applies if the initial deconfinement is much faster than the TADF processes and when the transfer between host sites is fast enough so that any deconfined triplet can quickly reach another guest site by diffusion. The diffusivity where this limit applies, therefore, also depends on the concentration. As will be shown in Sec. III, both processes should occur in the prompt regime.

When the intramolecular processes on the emitter molecules may be described using the three-level model, the rate equations that determine the volume densities $n_{\mathrm{S}}$ and $n_{\mathrm{T}}$ of singlet and triplet excitons, respectively, are in the fast-diffusion limit given by

$$
\begin{aligned}
\frac{\mathrm{d} n_{\mathrm{S}}}{\mathrm{d} t} & =-\left(k_{\mathrm{r}, \mathrm{S}}+k_{\mathrm{nr}, \mathrm{S}}+k_{\mathrm{ISC}}\right) n_{\mathrm{S}}+P_{\mathrm{T}, \mathrm{g}} k_{\mathrm{rISC}} n_{\mathrm{T}} \\
& \equiv-k_{\mathrm{S}} n_{\mathrm{S}}+P_{\mathrm{T}, \mathrm{g}} k_{\mathrm{rISC}} n_{\mathrm{T}}
\end{aligned}
$$

and

$$
\begin{aligned}
\frac{\mathrm{d} n_{\mathrm{T}}}{\mathrm{d} t} & =-P_{\mathrm{T}, \mathrm{g}}\left(k_{\mathrm{r}, \mathrm{T}}+k_{\mathrm{nr}, \mathrm{T}}+k_{\mathrm{rISC}}\right) n_{\mathrm{T}}+k_{\mathrm{ISC}} n_{\mathrm{S}} \\
& \equiv-P_{\mathrm{T}, \mathrm{g}} k_{\mathrm{T}} n_{\mathrm{T}}+k_{\mathrm{ISC}} n_{\mathrm{S}},
\end{aligned}
$$

where $k_{\mathrm{r}, \mathrm{S}(\mathrm{T})}$ and $k_{\mathrm{nr}, \mathrm{S}(\mathrm{T})}$ are the singlet (triplet) radiative and nonradiative decay rates of the TADF emitter, respectively, and $k_{\mathrm{ISC}}$ and $k_{\mathrm{rISC}}$ are the intersystem and reverse intersystem crossing rates of the TADF emitter, respectively (see Fig. 1). In the fast-diffusion limit, the effect of triplet deconfinement on the PL intensity decay is thus equivalent to that of a reduction of the rate coefficients $k_{\mathrm{r}, \mathrm{T}}$, $k_{\mathrm{nr}, \mathrm{T}}$, and $k_{\mathrm{rISC}}$ by a factor $P_{\mathrm{T}, \mathrm{g}}$. For a PL experiment with initially only singlets, and $n_{\mathrm{T}}(0)=0$, the solution of the coupled set of equations (2) and (3) is

$$
\begin{gathered}
n_{\mathrm{S}}(t) / n_{\mathrm{S}}(0)=A \exp \left(-k_{\mathrm{p}} t\right)+B \exp \left(-k_{\mathrm{d}} t\right), \\
n_{\mathrm{T}}(t) / n_{\mathrm{S}}(0)=-C \exp \left(-k_{\mathrm{p}} t\right)+C \exp \left(-k_{\mathrm{d}} t\right),
\end{gathered}
$$

with prompt (p) and delayed (d) decay rates that are given by

$$
k_{\mathrm{p}(\mathrm{d})}=\frac{1}{2}\left(k_{1} \pm \sqrt{k_{1}^{2}-k_{2}^{2}}\right)
$$

where

$$
\begin{gathered}
k_{1}=k_{\mathrm{S}}+P_{\mathrm{T}, \mathrm{g}} k_{\mathrm{T}}, \\
k_{2}=2 \sqrt{P_{\mathrm{T}, \mathrm{g}}\left(k_{\mathrm{S}} k_{\mathrm{T}}-k_{\mathrm{ISC}} k_{\mathrm{rIC}}\right)},
\end{gathered}
$$

and with

$$
\begin{gathered}
A=\frac{1}{2}\left[1+\frac{k_{\mathrm{S}}-P_{\mathrm{T}, \mathrm{g}} k_{\mathrm{T}}}{\sqrt{k_{1}^{2}-k_{2}^{2}}}\right], \\
B=\frac{1}{2}\left[1-\frac{k_{\mathrm{S}}-P_{\mathrm{T}, \mathrm{g}} k_{\mathrm{T}}}{\sqrt{k_{1}^{2}-k_{2}^{2}}}\right], \\
C=\frac{k_{\mathrm{ISC}}}{\sqrt{k_{1}^{2}-k_{2}^{2}}} .
\end{gathered}
$$

In a PL experiment, the recorded fluorescence signal is proportional to the singlet population. Therefore, the PL signal shows the TADF-characteristic bi-exponential decay that is described by Eq. (4). The fraction of excitons that decay during the prompt and delayed regime may be described using the integrated intensities from Eq. (4), such that

$$
\begin{aligned}
& \Phi_{\mathrm{PF}}=\int_{0}^{\infty} A \exp \left(-k_{\mathrm{p}} t\right)=\frac{A}{k_{\mathrm{p}}}, \\
& \Phi_{\mathrm{DF}}=\int_{0}^{\infty} B \exp \left(-k_{\mathrm{d}} t\right)=\frac{B}{k_{\mathrm{d}}} .
\end{aligned}
$$

As is common in the literature for experimentally studied systems, we will use the ratio of integrated intensities $\Phi_{\mathrm{DF}} / \Phi_{\mathrm{PF}}$ to quantify the TADF emission. ${ }^{21}$

Triplet-exciton deconfinement affects, in principle, the prompt as well as the delayed component. As an example, we consider materials for which $k_{\mathrm{nr}, \mathrm{S}}=k_{\mathrm{r}, \mathrm{T}}=k_{\mathrm{nr}, \mathrm{T}}=0$. Disregarding both rISC and deconfinement, the prompt rate $k_{\mathrm{p}}=k_{\mathrm{r}, \mathrm{S}}+k_{\mathrm{ISC}}$ is then determined only by radiative decay and ISC. Repopulation of the singlet state via fast rISC within the prompt regime reduces $k_{\mathrm{p}}$ [Eqs. (6)-(8)]. However, for most TADF emitters, $k_{\mathrm{p}}$ is not significantly affected by triplet upconversion or by triplet deconfinement. Often, to a good 
approximation, $k_{\mathrm{p}} \gg k_{\mathrm{d}}, k_{(\mathrm{n}) \mathrm{r}, \mathrm{S}} \gg k_{(\mathrm{n}) \mathrm{r}, \mathrm{T}}$, and $k_{\mathrm{ISC}} \gg k_{\mathrm{rISC}} \cdot{ }^{5}$ Under these conditions, $k_{\mathrm{p}} \approx k_{\mathrm{S}}$ and $k_{\mathrm{d}} \approx P_{\mathrm{T}, \mathrm{g}} \times\left(k_{\mathrm{T}}-k_{\mathrm{ISC}} k_{\mathrm{rISC}} / k_{\mathrm{S}}\right)$. In other words, the repopulation of singlets via rISC is then so slow that the prompt rate is to a good approximation equal to the sum of the radiative decay and ISC rates. As a result, the reduction of rISC by deconfinement then has no effect on the prompt component, but only on the decay in the delayed regime. With fast enough deconfinement and diffusion, $P_{\mathrm{T}, \mathrm{g}}$ [Eq. (1)] describes the relative fraction of the triplet population on guest molecules at all times. To what extent such fast exciton transfer is realistic will be discussed below.

Figure 3(a) shows the effect of deconfinement on the transient PL decay for a system containing $4 \mathrm{~mol} \%$ of a TADF emitter assuming equal DOS widths of the host and guest materials. We assumed that in the absence of deconfinement $k_{\mathrm{p}}=10^{8} \mathrm{~s}^{-1}$ and $k_{\mathrm{d}}=$ $10^{5} \mathrm{~s}^{-1}$ and the ratio of integrated intensities $\Phi_{\mathrm{DF}} / \Phi_{\mathrm{PF}}=10$, while $k_{\mathrm{nr}, \mathrm{S}}=k_{\mathrm{r}, \mathrm{T}}=k_{\mathrm{nr}, \mathrm{T}}=0$. The corresponding photophysical rates are $k_{\mathrm{r}, \mathrm{S}}=9.2 \times 10^{6} \mathrm{~s}^{-1}, k_{\mathrm{ISC}}=9.0 \times 10^{7} \mathrm{~s}^{-1}$, and $k_{\mathrm{rISC}}=1.1 \times 10^{6} \mathrm{~s}^{-1}$. The three parameters characterizing the PL decay are representative of state-of-the-art TADF emitters. Consistent with Fig. 2, we observe already for $\Delta E_{\mathrm{T}}$ smaller than $0.1 \mathrm{eV}$ a significant effect of deconfinement, leading to a lower intensity but a longer lifetime of the delayed component. As expected from the theoretical analysis, the prompt component is not significantly affected. Because no loss of triplets on the host is assumed, the integrated intensity remains constant, so that $\Phi_{\mathrm{DF}} / \Phi_{\mathrm{PF}}$ is independent of $\Delta E_{\mathrm{T}}$.

\section{B. Intermediate host diffusion}

The fast-diffusion conditions that were assumed in Sec. II A no longer apply if the diffusion time $t_{\text {diff }}$ needed to establish an equilibrium triplet occupancy over the entire host-guest system is larger than the transition time $t_{\mathrm{pd}}$ that marks the change from the prompt to the delayed decay regime. In such a case, the transfer of triplets to host molecules will lead to a decay curve that is in between that for the fast-diffusion limit and that for perfect confinement. Deconfinement will cease to play a role when the characteristic time for diffusion to the first nearest neighbor (NN) molecules, $t_{\text {diff,NN }}$, becomes larger than the characteristic time for the delayed decay, $t_{\mathrm{d}} \equiv k_{\mathrm{d}}^{-1}$. In this subsection, we investigate the role of deconfinement in the intermediate regime, for systems for which $\Delta E_{\mathrm{T}}=0 \mathrm{eV}$, with equal guest-host and host-host transfer rates and with $t_{\text {pd }} \ll t_{\text {diff,NN }}<t_{\text {diff }} \ll t_{\text {d. }}$. The deconfinement process then gives rise to an additional step in the intensity decay curve that occurs entirely within the delayed regime.

In the delayed regime, the relative density of singlets is very small and at its onset equal to the coefficient $B$, as given by Eq. (10). Almost all excitons in the system are triplets and the emission occurs due to relatively slow rISC, followed by relatively fast radiative singlet decay. When the triplet density is reduced due to diffusion to host sites, the singlet density is, therefore, reduced by the same factor. For the diffusion conditions mentioned above, and well within the time interval $t_{\text {diff,NN }}<t<t_{\text {diff, }}$, this reduction factor can be obtained using the standard continuum theory of three-dimensional diffusion from a point source (see Sec. S1 in the supplementary material). The relative density of singlets is then expected to be given by

$$
\frac{n_{\mathrm{S}}(t)}{n_{\mathrm{S}}(0)} \approx \frac{B a^{3}}{\left(4 \pi D_{\mathrm{h}} t\right)^{3 / 2}} \quad \text { for } t_{\mathrm{diff}, \mathrm{NN}}<t<t_{\mathrm{diff}}
$$

with $D_{\mathrm{h}}$ the host diffusion coefficient and $a$ the average intermolecular distance (defined such that there are $1 / a^{3}$ molecules per $\mathrm{m}^{3}$ ). In Fig. 3(b), we illustrate the effect for a material with a host diffusion coefficient $D_{\mathrm{h}}=3.2 \times 10^{-12} \mathrm{~m}^{2} \mathrm{~s}^{-1}$, which is a realistic value (see Sec. III B), and $a=1 \mathrm{~nm}$. The guest concentration is $4 \mathrm{~mol} \%$, as in Fig. 3(a), but all photophysical rates are taken a factor 10 larger in order to fulfill the requirement that the deconfinement process occurs entirely within the delayed emission regime. In the figure, the dashed line gives the singlet density that would follow
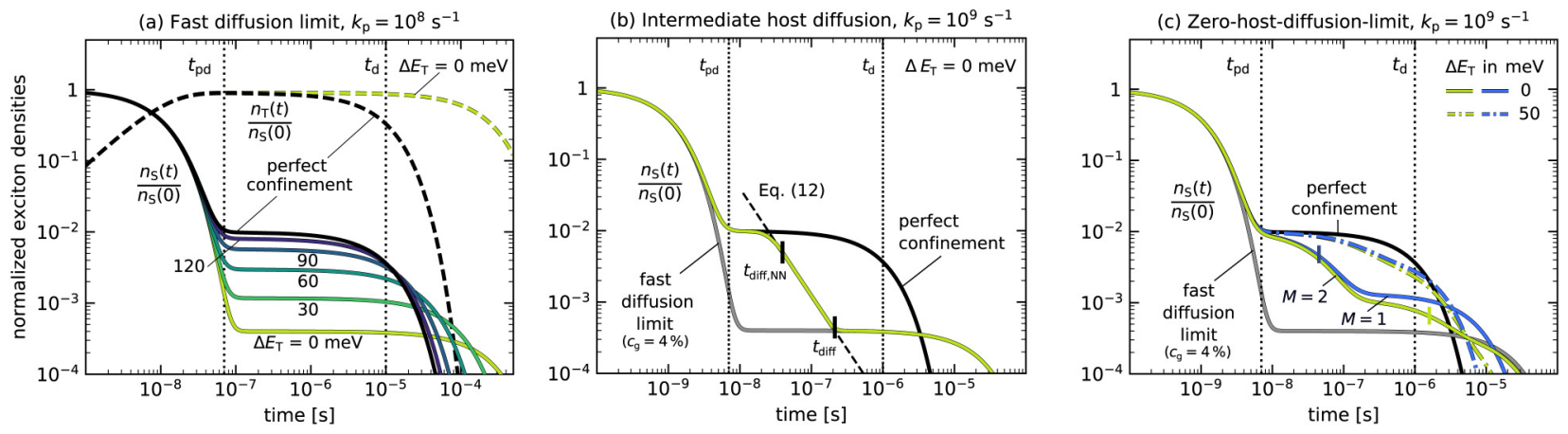

FIG. 3. Time dependence of the normalized exciton densities during a PL experiment for a $c_{g}=4 \%$ host-guest system. (a) Very fast triplet deconfinement and diffusion over the host, for various values of the triplet-exciton confinement energy. The photophysical rates are $k_{\mathrm{r}, \mathrm{S}}=9.2 \times 10^{6} \mathrm{~s}^{-1}, k_{\mathrm{ISC}}=9.0 \times 10^{7} \mathrm{~s}^{-1}$, and $k_{\mathrm{rISC}}=1.1 \times 10^{6} \mathrm{~s}^{-1}$. These rates are such that in the absence of deconfinement, $k_{\mathrm{p}}=10^{8} \mathrm{~s}^{-1}, k_{\mathrm{d}}=10^{5} \mathrm{~s}^{-1}$, and $\Phi_{\mathrm{DF}} / \Phi_{\mathrm{PF}}=10$. (b) Intermediate host diffusion with $D_{\mathrm{h}}=3.2 \times 10^{-12} \mathrm{~m}^{2} \mathrm{~s}^{-1}\left(k_{\mathrm{gh}}=k_{\mathrm{hh}}=3.2 \times 10^{6} \mathrm{~s}^{-1}\right)$, in a system with $\Delta E_{\mathrm{T}}=0 \mathrm{meV}$ and with tenfold enhanced photophysical rates as compared to (a). The four time scales indicated in the figure are defined in Sec. II B. (c) No host diffusion and deconfinement to the first and up to the second-nearest-neighbor molecules [blue ( $M=1$ ) and green $(M=2)$ curves, respectively, limit of low guest concentration], with $k_{\mathrm{gh}}=3.2 \times 10^{6} \mathrm{~s}^{-1}$ and with the same photophysical rates as in (b). The vertical bars indicate the inverse decay rates that follow from Eqs. (16) and (17), respectively. Results are shown for $\Delta E_{\mathrm{T}}=0$ (full curves) and $50 \mathrm{meV}$ (dashed-dotted curves). 
from Eq. (13) and the green curve gives the approximate overall intensity decay after including gradual transitions near the ends of the time range in which the deconfinement takes place. The figure shows that the deconfinement process occurs in a relatively narrow time range. The boundaries of that range may be obtained as follows. We define the times $t=t_{\text {diff,NN }}$ and $t=t_{\text {diff }}$ at which the deconfinement process starts and ends, respectively, as the times at which the singlet density has decreased by approximately a factor 2 and $1 / c_{\mathrm{g}}$, respectively. Within the framework of the continuum diffusion model leading to Eq. (13), it then follows that

$$
t_{\mathrm{diff}, \mathrm{NN}}^{*}=2^{2 / 3} \frac{a^{2}}{4 \pi D_{\mathrm{h}}} \quad \text { and } \quad t_{\mathrm{diff}}=\frac{1}{c_{\mathrm{g}}^{2 / 3}} \frac{a^{2}}{4 \pi D_{\mathrm{h}}} .
$$

We note that within the continuum model, no distinction is made between the transfer rates between like or unlike molecules. In actual systems, these can differ, so that the actual time at which the deconfinement process starts can deviate from the value given in Eq. (14). This is indicated by labeling this quantity of $t_{\text {diff,NN }}^{*}$ with an asterisk. In Sec. II C, we will develop an expression for $t_{\text {diff,NN }}$ that is based on the actual rate of guest-host transfer. For $4 \mathrm{~mol} \%$ systems, the ratio $t_{\text {diff }}^{*} / t_{\text {diff,NN }}$ is from Eq. (14) approximately equal to 5 .

\section{The zero-host-diffusion limit}

In the absence of host diffusion ("zero-host-diffusion limit"), the probability that a triplet resides on a guest molecule nonetheless decreases with time due to guest-host transfer. In this paper, we will treat the Dexter-type exciton transfer as a thermally activated hopping process with a rate that follows from the Miller-Abrahams (MA) formalism. ${ }^{22}$ For Dexter transfer between initial (i) and final (f) sites at a distance $R$ and with an energy difference $\Delta E=E_{\mathrm{f}}-E_{\mathrm{i}}$ the Dexter rate $k_{\mathrm{D}}$ is given by

$$
k_{\mathrm{D}}(R, \Delta E)=k_{\mathrm{D}, 1} \exp \left[-\frac{2(R-a)}{\lambda}\right] \exp \left[-\frac{|\Delta E|+\Delta E}{2 k_{\mathrm{B}} T}\right],
$$

with $k_{\mathrm{D}, 1}$ being the transfer rate between two equi-energetic nearest neighbor sites at a distance $a$ and $\lambda$ the exciton wavefunction decay length. Following a previous work, we take $\lambda=0.3 \mathrm{~nm}$ for the host and guest molecules. ${ }^{20}$

When the guest-host and guest-guest transfer rates are very fast and the concentration of guest molecules is large, a thermal equilibrium distribution of triplets on the guest and host states is quickly established so that the effect of these processes on the PL decay curve is equivalent to that of fast host diffusion. However, we find that for realistic values of the guest-host transfer rates and for dilute systems with realistic guest concentrations that this condition is not met (see Sec. III). Deconfinement due to transfer to neighboring host sites may be studied systematically and analytically by extending the set of rate equations for $n_{\mathrm{S}}$ and $n_{\mathrm{T}}$ [Eqs. (2) and (3)] to include transfer to and from host molecules at distinct distances, with weights that are determined by the radial distribution function. Within the framework of the MA formalism discussed above, the transfer rate from a guest molecule to a host molecule at a specific distance $R$ and triplet energy difference $\Delta E$ is determined by only a single parameter, $k_{\mathrm{D}, 1, \mathrm{gh}} \equiv k_{\mathrm{gh}}$.
In Fig. 3(c), we illustrate the effect by modeling the material as a simple cubic lattice with a lattice parameter $a=1 \mathrm{~nm}$. The full curves in the figures show for $\Delta E_{\mathrm{T}}=0 \mathrm{meV}$ the time-dependence of the singlet density, obtained analytically by solving a set of three or four coupled rate equations, for deconfinement to one of the six nearest-neighbor (NN) host molecules (blue curves) and up to the 12 second-nearest-neighbor (NNN) molecules (green curves), respectively. The dashed-dotted curves show the decay for $\Delta E_{\mathrm{T}}=50 \mathrm{meV}$. Including deconfinement up to the $M$ th nearestneighbor shell is found to lead to a normalized singlet density that is a sum of $M+2$ exponentially decaying functions. Initially, exciton deconfinement is only due to transfer to one of the NN molecules. Subsequently, also deconfinement to NNN molecules contributes. In a dilute system, successive deconfinement to ever more distant host sites occurs. The figure clearly shows sequential and well-separated NN and NNN deconfinement. Deconfinement to further neighbors is very slow, so that in the delayed emission regime, the singlet density does not reach the fast-diffusion limit level. The full curves have been obtained for the same parameters as used in (b), apart from switching-off exciton transfer between the host molecules and assuming a limit of low guest concentration. The role of guest concentration in the absence of host diffusion is demonstrated in Fig. S3 in the supplementary material.

We find that when the prompt, $\mathrm{NN}$, and $\mathrm{NNN}$ deconfinement and delayed decay rates are sufficiently different, the decay rate of the PL intensity due to deconfinement to the $N_{1}=6$ nearestneighbor molecules is equal to

$$
k_{\mathrm{NN}}=\left[1+N_{1} \exp \left(-\frac{\Delta E_{\mathrm{T}}}{k_{\mathrm{B}} T}\right)\right] k_{\mathrm{D}, 1, \mathrm{gh}} \equiv f_{1} k_{\mathrm{D}, 1, \mathrm{gh}},
$$

and the decay rate of the PL intensity due to deconfinement to the $N_{2}=12$ second-nearest-neighbor molecules is equal to

$$
k_{\mathrm{NNN}}=\left[\frac{1+\left(N_{1}+N_{2}\right) \exp \left(-\frac{\Delta E_{\mathrm{T}}}{k_{\mathrm{B}} T}\right)}{1+N_{1} \exp \left(-\frac{\Delta E_{\mathrm{T}}}{k_{\mathrm{B}} T}\right)}\right] k_{\mathrm{D}, 2, \mathrm{gh}} \equiv f_{2} k_{\mathrm{D}, 2, \mathrm{gh}},
$$

with $k_{\mathrm{D}, 2, \mathrm{gh}}$ the Dexter transfer rate to the NNN host molecules. In Fig. 3(c), the corresponding characteristic times are indicated by short vertical bars. The singlet density decreases by a factor $f_{1}$ and $f_{2}$, defined above, respectively. The additional decay due to deconfinement to more distant neighbor molecules can be calculated in an analogous manner, as long as the assumption that the successive deconfinement steps are sufficiently distinct and occur well in between the prompt and delayed decay. However, the effect of deconfinement to more distant neighbors is found to be relatively small, as is shown in Sec. S2 (Fig. S1) in the supplementary material.

It is of interest to compare the results of this microscopic model for deconfinement due to guest-host transfer with the continuum model developed in Sec. II B to describe the effect of host diffusion. For the case of diffusion due to $\mathrm{NN}$-only triplet transfer with a rate $k_{0}$ on a simple cubic lattice with site-independent triplet energies, the host diffusion coefficient is given by $D_{\mathrm{h}}=a^{2} k_{0}$. From Eq. (16), the onset time is then $1 / k_{\mathrm{NN}}=(1 / 7) k_{0}^{-1}$, which is very close to the time $t_{\text {diff,NN }}^{*} \approx 0.13 \times k_{0}^{-1}$ that follows from Eq. (14), 
i.e., from a continuum diffusion model within no distinction is made between guest-guest and host-host transfer.

We note that due to positional and energetic disorder the actual decay in this limit will be more gradual. Furthermore, the energetic disorder will give rise to a slightly reduced effective guest-host transfer rate. We expect that this reduction is similar to the reduction of the diffusion coefficient due to disorder, which from KMC simulations is found to be about a factor of four for disorder energies of $40 \mathrm{meV}$ (see Sec. III). We also note that within this analysis, the deconfinement from the guest sites are treated as independent processes. The analysis assumes thus that the guest concentration is sufficiently small, so that the host sites that are involved can only be reached from one guest site. The interference between deconfinement from nearby guest sites and the enhanced deconfinement due to host diffusion will be studied in Sec. III using KMC simulations.

\section{KINETIC MONTE CARLO SIMULATIONS}

In Sec. II, we have argued that the effect of triplet deconfinement depends (qualitatively) on the values of the times scale $t_{\text {diff,NN }}$ for NN transfer and $t_{\text {diff }}$ for host diffusion, relative to the characteristic times $t_{\mathrm{pd}}$ (prompt-delayed crossover) and $t_{\mathrm{d}}$ (delayed decay time) that follow from the perfect confinement curve. The value of $t_{\text {diff,NN }} \equiv 1 / k_{\mathrm{NN}}$ may be obtained from Eq. (16), while $t_{\text {diff }}$ may be obtained from Eq. (14). In Sec. II, we have already addressed the cases of $t_{\text {diff,NN }}$ being larger than $t_{\mathrm{d}}$ (perfect confinement) as well as $t_{\text {diff,NN }}$ and $t_{\text {diff }}$ both being smaller than $t_{\mathrm{pd}}$ (fast diffusion).

In this section, we first use the KMC simulation tool Bumblebee to establish the accuracy of the analytical models that have been presented in Sec. II. ${ }^{23}$ Next, we investigate the effect of triplet deconfinement for the case of directly competing time scales, namely, fast guest-host transfer $t_{\text {diff,NN }}<t_{\text {pd }}$ but weak or even no bulk diffusion, and with intermediate guest-host transfer $t_{\mathrm{pd}}<t_{\mathrm{diff}, \mathrm{NN}}<t_{\mathrm{d}}$ and intermediate to fast bulk diffusion. In Sec. III A, the simulation method is presented and applied to various limiting cases. The selection of realistic simulation parameters is motivated in Sec. III B, and the simulation results are given in Sec. III C. A comparison with experimental literature results is given in Sec. III D.

\section{A. Simulation method}

The organic semiconductor host-guest thin film is simulated as a $3 \mathrm{D}$ simple cubic lattice with a lattice constant $a$ of $1 \mathrm{~nm}$. Each site represents either a host or a guest molecule. The host and guest sites are randomly distributed with a molar guest concentration $c_{\mathrm{g}}$ and a host concentration $c_{\mathrm{h}}=1-c_{\mathrm{g}}$. The disorder of the film is taken into account by distributing the energy levels of the sites according to a Gaussian distribution. We assume equal standard deviations of the energetic disorder of the host and guest molecules, $\sigma_{\mathrm{g}}=\sigma_{\mathrm{h}}=40 \mathrm{meV}$, as used in a recent study of diffusion in phosphorescent host-guest systems. ${ }^{20}$

In addition to the intramolecular processes, shown in the left-hand side of Fig. 1, we include Dexter-type guest-host (gh), host-guest (hg), and host-host (hh) triplet transfer, as indicated in the right-hand side of Fig. 1. The transfer is treated as a thermally activated hopping process, with a rate that follows from the
Miller-Abrahams formalism that was already discussed in Sec. II C. ${ }^{22}$ For the sake of notational simplicity, the transfer rates to equi-energetic nearest-neighbor sites, $k_{\mathrm{D}, 1, \mathrm{gh}} \equiv k_{\mathrm{D}, 1, \mathrm{hg}}$ and $k_{\mathrm{D}, 1, \mathrm{hh}}$, will be written as $k_{\mathrm{gh}}$ and $k_{\mathrm{hh}}$, respectively. The resulting host diffusion coefficient, $D_{\mathrm{h}}$, is then given by

$$
D_{\mathrm{h}}=\alpha \gamma a^{2} k_{\mathrm{hh}}
$$

where $\alpha=1.295$ is an enhancement factor due to Dexter transfer to more remote neighbor molecules and $\gamma=0.239$ is a reduction factor due to the energetic disorder. In a previous work also, the dependence of these prefactors on the wavefunction decay length and the disorder energy has been discussed. ${ }^{20}$

The binding energy of the excitons is assumed to be sufficient to suppress all exciton dissociations. The simulations are performed in the low exciton density limit: at any moment, only a single exciton is present in the system. This implies that all excitonexciton annihilation interactions are excluded. Like in the analytical model, we assume that on the host molecules no radiative or nonradiative decay occurs. At each Monte Carlo step, transfer to all sites within a cube of side length $7 \mathrm{~nm}$, centered around the original triplet-exciton position, is included as a possible process. The simulations were carried out for systems with a size of $100 \times 100 \times$ 100 sites, with periodic boundary conditions. In order to obtain an appropriate statistical average we have carried out simulations for at least 20 of such systems, with different random disorder configurations. For each configuration, we have calculated the time at which radiative decay takes place for at least 3000 excitons, which at $t=0$ are randomly located on one of the guest molecules and in the singlet state. This assumes that in the experiment, the guest is either directly excited or that it is excited after very fast Förster-type transfer from a host molecule to a guest molecule. All simulations are carried out at room temperature $(T=300 \mathrm{~K})$.

We find that for the fast-diffusion limit as well as for the zero-host-diffusion limit, the KMC simulation results are consistent with the results from the analytical models for those limits. This is for the fast-diffusion limit demonstrated in Fig. S2(a) in the supplementary material, for systems with $D_{\mathrm{h}}=10^{-10} \mathrm{~m}^{2} \mathrm{~s}^{-1}$ containing TADF guest molecules with $k_{\mathrm{p}}=10^{8} \mathrm{~s}^{-1}, k_{\mathrm{d}}=10^{5} \mathrm{~s}^{-1}$, and $\Phi_{\mathrm{DF}} / \Phi_{\mathrm{PF}}=10$ at a concentration $c_{\mathrm{g}}=4 \mathrm{~mol} \%$. Figure S2(b) gives a comparison for systems with different host and guest disorder parameters. Figure S3 shows a comparison of KMC simulation results at $4 \mathrm{~mol} \%$ with analytical zero-host-diffusion results in the limit of low guest concentration, using the same photophysical rate parameters as in Fig. 3(c).

\section{B. Parameter value selection}

To study triplet-exciton deconfinement under realistic conditions, we need to determine the range of relevant exciton transfer rates in TADF host-guest systems. The experimental values of the triplet diffusion coefficient for various amorphous organic materials, including widely used host materials such as CBP and $\mathrm{mCP}$ (1,3-Bis(N-carbazolyl)benzene), are between $D_{\mathrm{h}}=10^{-13} \mathrm{~m}^{2} \mathrm{~s}^{-1}$ and $D_{\mathrm{h}}=10^{-10} \mathrm{~m}^{2} \mathrm{~s}^{-1} \cdot{ }^{20,24,25}$ From Eq. (18), the corresponding range of host-host triplet transfer rates is in the range of $k_{\mathrm{hh}}=3.2 \times 10^{5}-3.2 \times 10^{8} \mathrm{~s}^{-1}$. The reported triplet diffusion 
lengths are typically a few tens of nanometers, i.e., much larger than the average distance between the TADF emitter molecules. Deducing a range of typical values from available experimental information for $k_{\text {gh }}$ is at present quite difficult. We will, therefore, consider a wide range of values, similar to the range of values for $k_{\mathrm{hh}}$.

We remark that, in general, the Dexter transfer rate between unlike molecules in a mixture of two materials ( 1 and 2) with equal triplet-exciton wavefunction decay lengths can be estimated as the geometric mean $\sqrt{k_{1} k_{2}}$, with $k_{1}$ and $k_{2}$, the transfer rates that would follow from the bulk values of the materials' diffusion coefficients using Eq. (18). ${ }^{26}$ However, in view of the large ISC and rISC rates for TADF emitters, it is difficult to distinguish singlet and triplet diffusion. A detailed analysis of exciton diffusion in mixtures of the widely used emitter $4 \mathrm{Cz}$-IPN with the host material UGH2 by Menke and Holmes, taking the different diffusion behavior during the time spent in the singlet state and time spent in the triplet state into account, leads for pure $4 \mathrm{Cz}$-IPN to a nearestneighbor Dexter transfer rate $k_{\mathrm{D}, 1}(R=1 \mathrm{~nm}) \approx 8 \times 10^{7} \mathrm{~s}^{-1}$ and to an effective wavefunction decay length of $\lambda=2.5 \mathrm{~nm}$. $^{27}$ The authors suggest that the surprisingly large interaction range might be indicative of aggregation of the TADF emitter molecules when present in dilute systems, leading to a strongly reduced concentration dependence of the contribution of triplet diffusion to the total triplet diffusion length. In such a case also, analyses of exciton deconfinement should be adapted.

\section{Simulation results}

In Fig. 4, we show the results of KMC simulations regarding the sensitivity of the time-resolved PL intensity to deconfinement for various values of the host diffusion coefficient, guest concentration, guest-host transfer rate, and the photophysical rates of the guest molecules, for systems with $\Delta E_{\mathrm{T}}=0 \mathrm{eV}$. As indicated in the introduction of this section, we focus here on cases of deconfinement with competing timescales not yet covered in Sec. II.

Figures 4(a) and 4(b) show, for guest concentrations of 1 and $4 \mathrm{~mol} \%$, respectively, the sensitivity to the host diffusion coefficient for guests with photophysical rates as used in Fig. 3(a), and a large guest-host transfer rate, viz., $k_{\mathrm{gh}}=3.2 \times 10^{8} \mathrm{~s}^{-1}$. Host transfer with the same rate would lead to a relatively large diffusion coefficient, $D_{\mathrm{h}}=10^{-10} \mathrm{~m}^{2} \mathrm{~s}^{-1}$. The corresponding characteristic time for $\mathrm{NN}$ deconfinement is very small, viz., $t_{\text {diff } \mathrm{NN}} \sim 0.5 \mathrm{~ns}$ and thus well within the prompt regime. The intensity decay reveals even in the absence of host diffusion a considerable deviation from the perfect confinement curve. The zero-diffusion curves show around $10^{-6} \mathrm{~s}$ an accelerated intensity decrease, which from Fig. 3(c) (full $M=2$ curve) can be attributed to deconfinement to the second-nearest-neighbor host molecules. Due to the triplet energy disorder, which is included in the KMC simulations, this feature is less clearly pronounced than in the analytical model results. When the bulk diffusion is slow but finite $\left(D_{\mathrm{h}}=10^{-13} \mathrm{~m}^{2} \mathrm{~s}^{-1}\right)$, for the 1 and $4 \mathrm{~mol} \%$ systems according to Eq. (14), the deconfinement is expected to be complete at a time $t_{\text {diff }}$ of approximately 7 and $17 \mu \mathrm{s}$, respectively. The figures show that these are fair estimates and that the deconfinement completion times decrease inversely with $D_{\mathrm{h}}$, as expected.
Figure 4(c) shows in more detail the effect of a concentration variation on the intensity decay curves, for the photophysical and fast guest-host transfer rates used in panels (a) and (b) and for the case of a small host-diffusion coefficient $\left(D_{\mathrm{h}}=10^{-13} \mathrm{~m}^{2} \mathrm{~s}^{-1}\right)$. For the smallest guest concentration considered $(0.1 \mathrm{~mol} \%)$, the characteristic time $t_{\text {diff }}$ is about $8 \times 10^{-5} \mathrm{~s}$, which is much larger than $t_{\mathrm{d}}=1 \times 10^{-5} \mathrm{~s}$. However, due to the deconfinement, the end of the delayed emission regime is shifted, so that the fully deconfined state is nevertheless reached well before the end of the delayed emission.

Already at the beginning of the delayed emission regime, partial deconfinement has occurred due to NN guest-host transfer, whereas during the delayed emission regime, further deconfinement takes place due to a combination of guest-host transfer and bulk diffusion. On the other hand, for the largest concentration considered $(20 \mathrm{~mol} \%)$ the deconfinement process is almost entirely due to guest-host transfer. Although $t_{\text {diff }}$ is now much smaller $\left(\approx 2 \times 10^{-6} \mathrm{~s}\right)$, the figure shows that already much earlier the state of full deconfinement has been reached. The relative roles of deconfinement due to guest-host transfer and bulk diffusion depend thus strongly on the guest concentration.

Figure 4(d) gives simulation results for two emitters, at a concentration of $4 \mathrm{~mol} \%$, with the sets of photophysical rates of the guest that were used in Fig. 3(a) (green curves and symbols) and with tenfold larger photophysical rates (blue curves and symbols), as used in Figs. 3(b) and 3(c). The host diffusion coefficient is taken large $\left(D_{\mathrm{h}}=10^{-10} \mathrm{~m}^{2} \mathrm{~s}^{-1}\right.$, corresponding to $\left.k_{\mathrm{hh}}=3.2 \times 10^{8} \mathrm{~s}\right)$. As the value of $t_{\text {diff }} \approx 7 \times 10^{-9} \mathrm{~s}$ is for both emitters smaller than $t_{\mathrm{pd}}$, the role of deconfinement is for both emitters determined by the guest-host transfer rate. That rate is varied from a value equal to $k_{\mathrm{hh}}$ to a three orders of magnitude lower value. As expected, the figure reveals a strong effect of such a decrease of $k_{\mathrm{gh}}$. When the deconfinement occurs in the delayed regime, it is seen to lead to an intermediate step in the decay curve in a time range that shifts proportionally to $k_{\mathrm{gh}}$. Host diffusion is expected to determine the shape of the step. Close inspection of the simulation curves shows that, consistent with Eq. (13), the intensity decays indeed to a good approximation proportionally to $t^{-3 / 2}$. The figure also shows that the emission curves are then quite independent of the photophysical rates. To a fair approximation, that happens for $k_{\mathrm{gh}}$ in the range $3.2 \times\left(10^{5}-10^{6}\right) \mathrm{s}^{-1}$. This observation validates our general picture, within which the characteristic times $t_{\text {diff,NN }}$ an $t_{\text {diff }}$ that determine the shape of the decay curves in the delayed regime are independent of the photophysical rates when both time constants fall within the delayed regime.

The KMC simulations show qualitatively similar effects on the delayed emission for materials with positive values of $\Delta E_{\mathrm{T}}$, as shown in Fig. S4 in the supplementary material for $4 \mathrm{~mol} \%$ systems. The effect on the PL decay becomes then negligible for $\Delta E_{\mathrm{T}}>0.1 \mathrm{eV}$, consistent with the effect shown in Fig. 3(a).

\section{Comparison with experiment}

The analytical and KMC simulation results show that to achieve good confinement in systems with guest concentrations down to $1 \mathrm{~mol} \%$ a triplet confinement energy of $\Delta E_{\mathrm{T}} \geq 0.2 \mathrm{eV}$ is needed. The fraction of triplets that stays on the guest molecules is then always larger than $95 \%$. This criterion for good confinement 
(a) Varying $D_{\mathrm{h}}$ at $c_{\mathrm{g}}=1 \%$

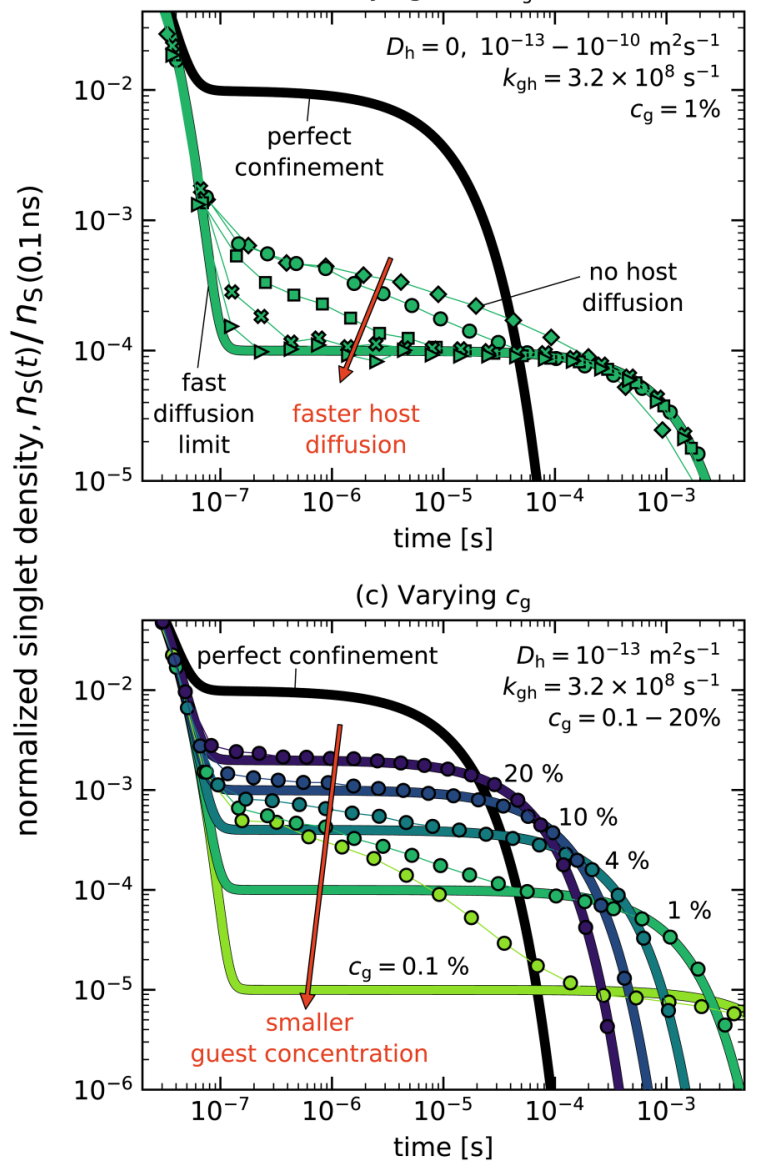

(b) Varying $D_{\mathrm{h}}$ at $c_{\mathrm{g}}=4 \%$

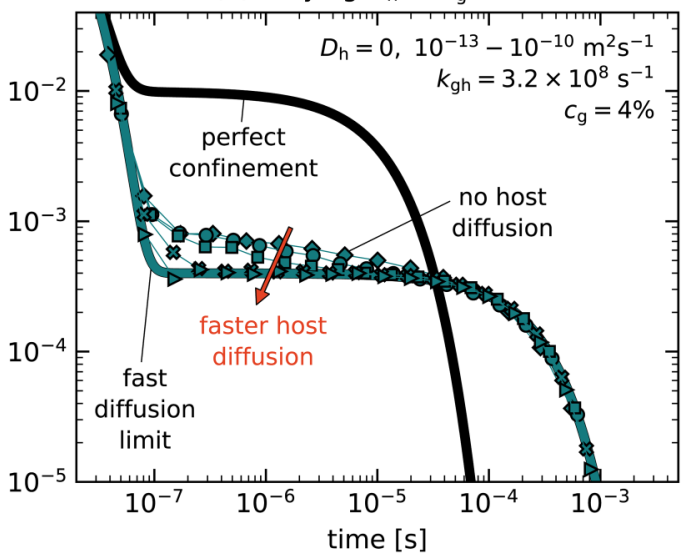

(d) Varying $k_{\mathrm{gh}}$ at different $k_{\mathrm{p}}$

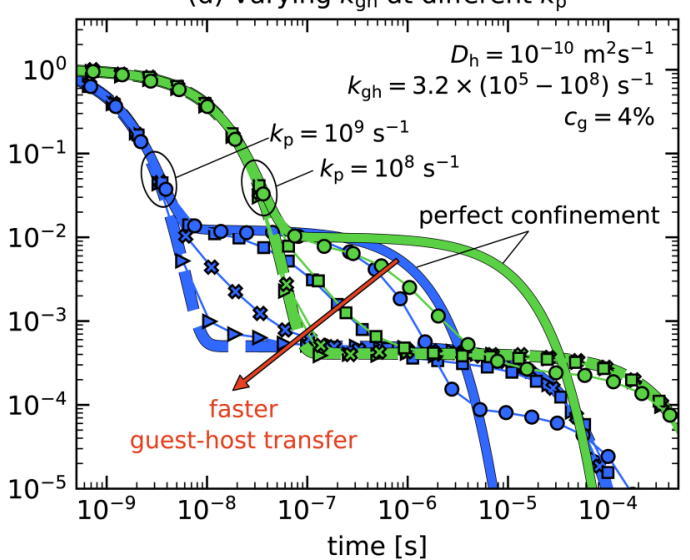

FIG. 4. Time dependence of the normalized singlet exciton density for various values of the host diffusion coefficient, guest concentration, guest-host transfer rate, and photophysical rates of the guest molecules, for systems with $\Delta E_{\mathrm{T}}=0 \mathrm{eV}$. The full curves show analytical solutions for the perfect confinement and fast-diffusion limits, and the symbols show KMC simulation results. (a) Dependence on the host diffusion coefficient, varied in steps of a factor 10, for guests with photophysical rates as used in Fig. 3(a), for guest-host transfer rate of $k_{\text {gh }}=3.2 \times 10^{8} \mathrm{~s}^{-1}$, at a guest concentration of $c_{\mathrm{g}}=1 \mathrm{~mol} \%$. (b) As in (a), for a guest concentration of 4 mol \%. (c) Dependence on the guest concentration as indicated in the figure, for a small host diffusion coefficient of $D_{\mathrm{h}}=10^{-13} \mathrm{~m}^{2} \mathrm{~s}^{-1}$, large $k_{\text {gh }}=3.2 \times 10^{8} \mathrm{~s}^{-1}$ and for the photophysical rates as used in (a). (d) Dependence on the guest-host transfer rate, varied in steps of a factor 10, for guests with photophysical rates as used in Fig. 3(a) (green curves and symbols, $k_{p}=1 \times 10^{8} \mathrm{~s}^{-1}$ ) and Figs. $3(\mathrm{~b})$ and $3(\mathrm{c})$ (blue curves and symbols, $k_{\mathrm{p}}=1 \times 10^{9} \mathrm{~s}^{-1}$ ), for $c_{\mathrm{g}}=4 \mathrm{~mol} \%$.

is similar to that for triplet deconfinement from Ir-cored phosphorescent emitter molecules to many host molecules, as obtained experimentally from electroluminescence, photoluminescence, and triplet-triplet annihilation studies. ${ }^{28-31}$ The effect of deconfinement on the PL decay decreases with increasing guest concentration, so that for $c_{\mathrm{g}} \geq 20 \%$, the same exciton occupation on guest sites can already be achieved for $\Delta E_{\mathrm{T}} \geq 0.1 \mathrm{eV}$.

Experimentally validating the criterion for good confinement in TADF host-guest systems is not straightforward. Within the framework of our modeling study, the total PL efficiency is formally expected to be equal to that of singlets on the TADF emitter and independent of $\Delta E_{\mathrm{T}}$. One might ask how in practical systems, the total PL efficiency varies with $\Delta E_{\mathrm{T}}$. Such experiments have been performed by Sato et al. for TADF host-guest systems based on the TADF emitter 2-biphenyl-4,6-bis(12-phenylindolo[2,3-a] carbazole-11-yl)-1,3,5-triazine (PIC-TRZ2), with a triplet energy of about $2.6 \mathrm{eV}{ }^{19}$ Even when embedding the emitter in materials with triplet energies above up to $2.8 \mathrm{eV}$, the PL efficiency was found to show a tendency to increase with increasing $\Delta E_{\mathrm{T}}$, up to a triplet energy of about $3.5 \mathrm{eV}$. Our study shows that this effect cannot be related to triplet deconfinement. Alternatively, it could be due to an effect of the host on the non-radiative decay rate of triplets on the TADF molecule, as suggested by the authors for the host (UGH2) with the largest triplet energy included in their study. Further timeresolved measurements would be needed to disentangle the various effects. As expected, triplet deconfinement is observed in hosts with insufficient $\Delta E_{\mathrm{T}}$ and leads to an efficiency loss due to non-radiative decay on the host. 
Experimental studies have shown that the PL intensity of TADF systems can show at long time scales a tail following a power-law behavior. ${ }^{7,32}$ From our analysis, we expect in limited time intervals, a power-law decrease with a slope of $-3 / 2$ when bulk diffusion determines the deconfinement rate [Fig. 3(b)] and under some conditions a decrease with a smaller slope [e.g., Fig. 4(a)]. In the studies cited, the slope is somewhat larger and smaller, respectively. This is an indication of complicating effects that have not been included in our study, such as (non-)radiative decay of triplets on host molecules, a distribution of photophysical rates due to conformational disorder, and a time-dependent singlet-triplet gap due to a time-dependent polarization of the host molecules towards the emitters. ${ }^{21,32,33}$

\section{SUMMARY AND CONCLUSIONS}

Using analytical modeling and KMC simulations, we have studied how triplet deconfinement in TADF host-guest systems as employed in OLEDs is expected to affect the transient photoluminescence from these materials. In our description of the kinetics of the deconfinement process, we have made a distinction between the transfer rate from the emissive guest molecules to the nearestneighbor (NN) host molecules and the triplet diffusion over the host materials, determined by the transfer rate between pairs of host molecules. We find that the shape of the decay curve is ultimately determined by the interplay of the characteristic times $t_{\text {diff,NN }}$ and $t_{\text {diff }}$ for these two processes and their relation to the transition time $t_{\mathrm{pd}}$ between TADF regimes, as well as the delayed decay time $t_{\mathrm{d}}$.

In the fast-diffusion limit, $t_{\text {diff,NN }}$ and $t_{\text {diff }}$ are both smaller than $t_{\mathrm{pd}}$. Independent of the precise transfer rates, the PL decay is then determined by the thermodynamic occupation probability of triplet excitons on the host and guest molecules. For that limit, analytical expressions are given. These show that for $1 \mathrm{~mol} \%$ systems, triplet-exciton deconfinement starts to affect the transient PL intensity curves when the triplet confinement energy becomes smaller than $\Delta E_{\mathrm{T}} \sim 0.2 \mathrm{eV}$. For $10 \mathrm{~mol} \%$ systems, this occurs for $\Delta E_{\mathrm{T}}$ below approximately $0.1 \mathrm{eV}$. Notably, the transient PL intensity decay in this fast-diffusion limit remains bi-exponential, albeit the delayed intensity being reduced and the delayed decay time enhanced. Therefore, deconfinement can in principle not be ruled out by a single PL measurement. In the study of TADF materials, consistently and comparably extracting the photophysical parameters, such as the ISC and rISC rates, is a vital tool and identifying and suppressing deconfinement is one of the necessary components to do so. ${ }^{34}$

For rates that realistically describe the guest-host and hosthost triplet transfer, and for realistic photophysical rates of the TADF molecules, we find that the PL decay curve can be more complex, e.g., showing in the delayed regime $\left(t_{\mathrm{pd}}<t<t_{\mathrm{d}}\right)$ a distinct additional intensity decrease, caused by host diffusion and being proportional to $t^{-3 / 2}$. In the case of very weak host diffusion but sufficiently fast guest-host transfer, this additional intensity decrease is expected to reflect the pair distribution function, i.e., the distribution of distances to the nearest-neighbor and more distant molecules. The multistep-shaped decrease that we have obtained for the case of a simple cubic lattice will in realistic materials be smeared out due to positional and transfer-integral disorder. KMC studies show that also when $t_{\text {diff,NN }}$ and $t_{\text {diff }}$ do not fall in the delayed regime, the shape of the PL curves can be rationalized on the basis of the four characteristic time scales. A variation of the guest concentration, which only affects $t_{\text {diff }}$, is expected to provide a sensitive method for separating the contributions of guest-host transfer and host diffusion.

\section{SUPPLEMENTARY MATERIAL}

The supplementary material contains a derivation of the point-source diffusion model used in Sec. II B (S1), the timedependence of $P_{\mathrm{T}, \mathrm{g}}$ in the case of host-guest transfer without host diffusion (S2), a comparison of the analytical model results with $\mathrm{KMC}$ results (S3), and KMC simulation results demonstrating the interplay of different confinement energies $\Delta E_{\mathrm{T}}$ and host-guest transfer rates $k_{\mathrm{gh}}$.

\section{ACKNOWLEDGMENTS}

This project received funding from the European Union Horizon 2020 research and innovation programme under the Marie Skłodowska-Curie Grant Agreement No. 812872 (TADFlife). The authors thank A. Ligthart and X. de Vries for useful discussions and $\mathrm{M}$. Barbry for technical support.

\section{DATA AVAILABILITY}

The data that support the findings of this study are available from the corresponding author upon reasonable request.

\section{REFERENCES}

${ }^{1}$ H. Uoyama, K. Goushi, K. Shizu, H. Nomura, and C. Adachi, Nature 492, 234 (2012).

${ }^{2}$ C. Adachi, Jpn. J. Appl. Phys. 53, 060101 (2014).

${ }^{3}$ H. Kaji, H. Suzuki, T. Fukushima, K. Shizu, K. Suzuki, S. Kubo, T. Kimono, H. Oiwa, F. Suzuki, A. Wakamiya, Y. Murata, and C. Adachi, Nat. Commun. 6, 8476 (2015).

${ }^{4}$ M. N. Berberan-Santos and J. M. M. Garcia, J. Am. Chem. Soc. 118, 9391 (1996).

${ }^{\mathbf{5}}$ K. Goushi, K. Yoshida, K. Sato, and C. Adachi, Nat. Photonics 6, 253 (2012).

${ }^{6}$ L. Bergmann, G. J. Hedley, T. Baumann, S. Bräse, and I. D. W. Samuel, Sci. Adv. 2, e1500889 (2016).

${ }^{7}$ N. Haase, A. Danos, C. Pflumm, A. Morherr, P. Stachelek, A. Mekic, W. Brütting, and A. P. Monkman, J. Phys. Chem. C 122, 29173 (2018).

${ }^{8}$ B. Yurash, H. Nakanotani, Y. Olivier, D. Beljonne, C. Adachi, and T. Nguyen, Adv. Mater. 31, 1804490 (2019).

${ }^{9}$ S. Gottardi, M. Barbry, R. Coehoorn, and H. van Eersel, Appl. Phys. Lett. 114, 073301 (2019).

${ }^{10}$ T. Kobayashi, A. Niwa, S. Haseyama, K. Takaki, T. Nagase, K. Goushi, C. Adachi, and H. Naito, J. Photonics Energy 8, 032104 (2018).

${ }^{11}$ T. J. Penfold, F. B. Dias, and A. P. Monkman, Chem. Comm. 54, 3926 (2018).

${ }^{12}$ W. Li, Y. Pan, L. Yao, H. Liu, S. Zhang, C. Wang, F. Shen, P. Lu, B. Yang, and Y. Ma, Adv. Opt. Mater. 2, 892 (2014).

${ }^{13}$ S. Zhang, L. Yao, Q. Peng, W. Li, Y. Pan, R. Xiao, Y. Gao, C. Gu, Z. Wang, P. Lu, F. Li, S. Su, B. Yang, and Y. Ma, Adv. Funct. Mater. 25, 1755 (2015).

${ }^{14}$ Y. Pan, J. Huang, W. Li, Y. Gao, Z. Wang, D. Yu, B. Yang, and Y. Ma, RSC Adv. 7, 19576 (2017).

${ }^{15}$ H. Sun, Z. Hu, C. Zhong, X. Chen, Z. Sun, and J.-L. Brédas, J. Phys. Chem. Lett. 8, 2393 (2017). 
${ }^{16}$ C. Deng, L. Zhang, D. Wang, T. Tsuboi, and Q. Zhang, Adv. Opt. Mater. 7, 1801844 (2019).

${ }^{17}$ G. Méhes, K. Goushi, W. J. Potscavage, and C. Adachi, Org. Electron. 15, 2027 (2014).

${ }^{18}$ N. Notsuka, R. Kabe, K. Goushi, and C. Adachi, Adv. Funct. Mater. 27, 1703902 (2017).

${ }^{19}$ K. Sato, K. Shizu, K. Yoshimura, A. Kawada, H. Miyazaki, and C. Adachi, Phys. Rev. Lett. 110, 247401 (2013).

${ }^{20}$ A. Ligthart, X. de Vries, P. A. Bobbert, and R. Coehoorn, Org. Electron. 77, 105510 (2020).

${ }^{21}$ F. B. Dias, J. Santos, D. R. Graves, P. Data, R. S. Nobuyasu, M. A. Fox, A. S. Batsanov, T. Palmeira, M. N. Berberan-Santos, M. R. Bryce, and A. P. Monkman, Adv. Sci. 3, 1600080 (2016).

${ }^{22}$ A. Miller and E. Abrahams, Phys. Rev. 120, 745 (1960).

${ }^{23}$ The bumblebee software is provided by Simbeyond B.V. See https://simbeyond.com.

${ }^{24}$ T. Chatterjee and K.-T. Wong, Adv. Opt. Mater. 7, 1800565 (2019).

${ }^{25}$ O. V. Mikhnenko, P. W. M. Blom, and T.-Q. Nguyen, Energy Environ. Sci. 8, 1867 (2015).
${ }^{26}$ M. Mesta, M. Carvelli, R. J. de Vries, H. van Eersel, J. J. M. van der Holst, M. Schober, M. Furno, B. Lüssem, K. Leo, P. Loebl, R. Coehoorn, and P. A. Bobbert, Nat. Mater. 12, 652 (2013).

${ }^{27}$ S. M. Menke and R. J. Holmes, J. Phys. Chem. C 120, 8502 (2016).

${ }^{28}$ R. J. Holmes, S. R. Forrest, Y.-J. Tung, R. C. Kwong, J. J. Brown, S. Garon, and M. E. Thompson, Appl. Phys. Lett. 82, 2422 (2003).

${ }^{29}$ K. Goushi, R. Kwong, J. J. Brown, H. Sasabe, and C. Adachi, J. Appl. Phys. 95, 7798 (2004).

${ }^{30}$ Y. Kawamura, K. Goushi, J. Brooks, J. J. Brown, H. Sasabe, and C. Adachi, Appl. Phys. Lett. 86, 071104 (2005).

${ }^{31}$ A. Ligthart, X. de Vries, L. Zhang, M. C. W. M. Pols, P. A. Bobbert, H. van Eersel, and R. Coehoorn, Adv. Funct. Mater. 28, 1804618 (2018).

${ }^{32}$ P. L. dos Santos, J. S. Ward, P. Data, A. S. Batsanov, M. R. Bryce, F. B. Dias, and A. P. Monkman, J. Mater. Chem. C 4, 3815 (2016).

${ }^{33}$ Y. Olivier, B. Yurash, L. Muccioli, G. D'Avino, O. Mikhnenko, J. C. Sancho-García, C. Adachi, T.-Q. Nguyen, and D. Beljonne, Phys. Rev. Mater. 1, 075602 (2017).

${ }^{34}$ M. Y. Wong and E. Zysman-Colman, Adv. Mater. 29, 1605444 (2017). 